\title{
Cyclodextrin-grafted cellulose: Physico-chemical characterization
}

\author{
B. Medronho a,b,*, R. Andrade ${ }^{a}, V^{2}$ Vivod $^{c}$, A. Ostlund ${ }^{d}$, M.G. Miguel $^{a}$, B. Lindman $^{\mathrm{a}, \mathrm{e}}$, \\ B. Voncina ${ }^{\mathrm{c}}$, A.J.M. Valente ${ }^{\mathrm{a}}$ \\ a University of Coimbra, Department of Chemistry, Coimbra, Portugal \\ ${ }^{\mathrm{b}}$ University of Algarve, Laboratory of Plant Biotechnology, Campus de Gambelas, Faro, Portugal \\ c University of Maribor, Faculty of Mechanical Engineering, Department of Textile Materials and Design, Smetanova 17, 2000 Maribor, Slovenia \\ d Applied Surface Chemistry, Department of Chemical and Biological Engineering, Chalmers University of Technology, 41296 Göteborg, Sweden \\ e Division of Physical Chemistry, Center of Chemistry and Chemical Engineering, Lund University, Sweden
}

\section{A R T I C L E I N F O}

\section{Article history:}

Received 25 October 2011

Received in revised form 22 May 2012

Accepted 28 August 2012

Available online 5 September 2012

\section{Keywords:}

Cellulose

Cyclodextrin

Polycarboxylic acid

Dissolution

FTIR

\begin{abstract}
A B S T R A C T
Cyclodextrins (CDs) can form inclusion complexes with a wide variety of molecules making them very attractive in different areas, such as pharmaceutics, biochemistry, food chemistry and textile. In this communication we will report on the physico-chemical characterization of cellulose modified with CDs by means of infra-red spectroscopy (FTIR), cross polarization magic angle spinning solid state nuclear magnetic resonance (CP-MAS NMR), polarized optical microscopy (POM) and thermal gravimetric analysis (TGA). Both CP-MAS NMR and FTIR indicate that CDs are chemically attached to cellulose backbone through the formation of ester bonds. Furthermore, the CD-grafted cellulose was dissolved in a "superphosphoric" acid solution but, despite the increase of hydrophilicity due to the modification, POM revealed that grafted cellulose was less soluble when compared to the unmodified polymer. The formation of a complex CD-cellulose network is suggested.
\end{abstract}

(c) 2012 Elsevier Ltd. All rights reserved.

\section{Introduction}

Cyclodextrins (CDs) are cyclic oligosaccharides with a truncated cone shape containing 5 or more D-glucopyranose units netted through covalent bonds by C1 and C4 carbons (Li \& Purdy, 1992; Saenger et al., 1998; Uekama, Hirayama, \& Irie, 1998; Vogtle, 1991). By far, beta-cyclodextrin, with 7 sugar units, has been the most commercially attractive (more than $95 \%$ of CDs produced and consumed) due to its simple synthesis, availability and price. The cavity is lined by the hydrogen atoms and the glycosidic oxygen bridges. The nonbonding electron pairs of the glycosidic oxygen bridges are directed toward the inside of the cavity, producing a high electron density and lending it some Lewis base character. As a result of this spatial arrangement of the functional groups in the CDs molecules, the cavity is relatively hydrophobic while the external surfaces are hydrophilic (Del Valle, 2004). CDs can form inclusion complexes with a wide variety of molecules. Such interactions result in an alteration of the physical properties of the solutions, and these properties have been used in areas as diverse as encapsulation of active substances (i.e. such as flavoring agents, metallic cations, fragrances and pesticides) (Martel, Morcellet, Ruffin, Ducoroy, \& Weltrowski, 2002; Martel, Weltrowski, Ruffin, \& Morcellet, 2002;

\footnotetext{
* Corresponding author at: University of Algarve, Laboratory of Plant Biotechnology, Campus de Gambelas, Faro, Portugal. Tel.: +351 289800910x7910.

E-mail address: bfmedronho@portugalmail.pt (B. Medronho).
}

Martel, Ruffin, Weltrowski, Lekchiri, \& Morcellet, 2005; Martel, Ducoroy, Bacquet, \& Morcellet, 2007; Martel, Ducoroy, Bacquet, \& Morcellet, 2007), enzymatic synthesis (Zelinski, Liese, Wandrey, \& Kula, 1999), drug delivery (Guyot, Fawaz, Bildet, Bonini, \& Lagueny, 1995; Murthy \& Shown, 2008; Uekama et al., 1998; Utsuki et al., 1996), catalysis (Natoli, Pagliero, Trotta, \& Drioli, 1997) and energy transfer studies (Lis, Elbanowski, Makowska, \& Hnatejko, 2002). The considerable increase of solubility of a guest molecule in aqueous media, its protection against oxidation and thermal or chemical degradation, and prevention of rapid evaporation of volatile guest compounds are a few examples where CDs performance stands out (Szejtli, 2003).

Cellulose is the most abundant natural polymer on earth. It is virtually an inexhaustible source of raw material for environmentally friendly (non-toxic), biocompatible, renewable and modifiable polymers which makes it one of the most promising feedstocks for industry in the future. Production of cellulose based fibers might become the only sustainable future alternative to cotton when new processes for dissolution and fiber spinning of cellulose are developed (Lindman, Karlstrom, \& Stigsson, 2010). In order to increase the functionality of cellulose and produce so called "smart" fibers, synthesis procedures have been developed. In fact, the modification and functionalization of natural fibers with CDs is being implemented in modern textile processing. Physical (impregnation) or chemical (covalent binding) interaction of CDs with textiles can assign to the latter the aforementioned complexforming ability of CDs and can represent new functionalities for 
the textile supports, new possibilities of applications and new markets to explore. For the last 30 years, the use of cyclodextrins and their derivatives in the textile domain has captivated a lot of attention (Buschmann, Knittel, Beermann, \& Schollmeyer, 2001; Buschmann, Knittel, \& Schollmeyer, 2001; Buschmann, 2001; Denter \& Schollmeyer, 1996; Murthy \& Shown, 2009; Szejtli, 2003). Many of the papers and patents report the use of CDs for finishing and dyeing processes (Savarino, Viscardi, Quagliotto, Montoneri, \& Barni, 1999; Voncina, Vivod, \& Jausovec, 2007). Others either discuss, for instance, the capture of unpleasant smells due to perspiration (Buschmann, Knittel, \& Schollmeyer, 1991; Poulakis, Buschmann, \& Schollmeyer, 2002), or how to do the controlled release of perfumes (Fujimura, 1985; Ritter, Delney, Volz, \& Kerr, 2002), insecticides (Akasaka, Shibata, \& Ochia, 1991; Akasaka, Sawai, Iwase, \& Moriishi, 1992) and antibacterial agents (Voncina \& Majcen, 2004; Yamamoto \& Saeki, 1998).

The grafting of CDs onto natural and synthetic fibers has first been reported by Szejtli et al. (Szejtli, Zsadon, Fenyvesi, Horvath, \& Tudos, 1980) which, via a condensation reaction using epichlorohydrin as a crosslinking agent, were able to graft CDs onto cellulose fibers (cotton). Poulakis et al. (Poulakis, Buschmann, \& Schollmeyer, 1992) reported the physical and chemical incorporation of CDs into natural or synthetic matter. In a similar way, Buschman et al. (Buschman, Denter, Knittel, \& Schollmeyer, 1998) claimed the incorporation of $\mathrm{CDs}$ into natural or synthetic materials by physical means or by chemical paths involving $C D$ derivatives carrying aliphatic and aromatic groups, chlorocarboxylic acids, and chloroamino and dimethylol bifunctional compounds as linking agents. Denter et al. (Denter \& Schollmeyer, 1996; Denter, Buschmann, Knittel, \& Schollmeyer, 1997) have used cyanuric chloride as a crosslinking agent and Reuscher and coworkers (Reuscher, Hirsenkorn, \& Haas, 1995) have fixed a monochlorotriazinyl CD derivative onto different polymer materials, including cotton fibers. Furthermore, in a recent study, Martel et al. (Martel, Morcellet, et al., 2002; Martel, Weltrowski, et al., 2002) grafted CDs permanently onto polypropylene fabrics and wool fibers by electron beam techniques and using poly(carboxylic) acids (PCAs) as crosslinking agents. The method used found inspiration in the work done by Welch (1988) where a PCA family of compounds was used in the crosslinking reaction of cotton applied to the durable press finishing processing. Indeed the use of PCAs, such as 1,2,3,4-butane tetracarboxylic acid (BTCA) are well known non-formaldehyde crosslinking reagents (Gagliardi \& Shippee, 1963; Voncina \& Le Marechal, 2005). BTCA has four carboxylic acid groups, which can react with hydroxyl groups of cellulose and form stable ester bonds (Martel, Morcellet, et al., 2002; Martel, Weltrowski, et al., 2002; Morris, Catalano, \& Andrews, 1995). Rowland et al. (Rowland, 1968; Rowland, Welch, Brannan, \& Gallagher, 1967) studied in detail the esterification reactions of PCA with cotton cellulose and the indication was that high levels of cellulose crosslinking could be obtained with PCA with three or more carboxyl groups per molecule. Esterification can occur with heat alone or can be accelerated by the presence of salts of weak acids, such as sodium hypophosphite (SHPI). In this paper, we revisit the parameters that control the CD grafting onto a cellulose surface and characterize the modified polymer by means of FTIR spectroscopy, TGA, and solid state NMR. Moreover, we evaluate the possibility of dissolution and spinning of fibers from the new functionalized cellulose material.

\section{Experimental}

\subsection{Materials}

$\alpha$-Cellulose with a molecular weight of ca. $212 \mathrm{~kg} / \mathrm{mol}$ (corresponding to a degree of polymerization of around 1780) was of
Table 1

Initial conditions for the synthesis process of grafting of $\beta$-cyclodextrin on $\alpha$ cellulose. Units are given in wt\%.

\begin{tabular}{lllll}
\hline Sample & $\beta-\mathrm{CD}(\%)$ & BTCA $(\%)$ & Catalyst $(\%)$ & Weight gain $(\%)$ \\
\hline A & 8 & 6 & 3 & 32.4 \\
B & 4 & 6 & 3 & 12.9 \\
C & 8 & 3 & 3 & 10.0 \\
\hline
\end{tabular}

analytical grade and purchased from Sigma Aldrich. $\beta$-cyclodextrin was also purchased from Sigma Aldrich with a water content of $13.1 \mathrm{wt} \%$ (molar ratio of $\mathrm{H}_{2} \mathrm{O}$ to $\mathrm{CD}$ is $17: 2$ ) as determined from thermal analysis. 1,2,3,4-butanetetracarboxylic acid (BTCA, 98\%) and sodium hypophosphite-1-hydrate (SHPI, 99\%) were supplied by Acros Organics and Riedel-de Haen, respectively. Sodium hydroxide (p.a., $>98 \%$ ) and polyphosphoric acid $\left(>83 \% \mathrm{P}_{2} \mathrm{O}_{5}\right)$ were provided by Sigma-Aldrich.

\subsection{Grafting of $\alpha$-cellulose with $\beta$-cyclodextrin}

$\alpha$-Cellulose was immersed in treating baths containing $\beta-C D$ and BTCA and to reduce the curing temperature the catalyst SHPI was used (concentrations of reagents are shown in Table 1). $\mathrm{pH}$ values of all treating baths were 2.7. All impregnated samples were pre-dried at $110^{\circ} \mathrm{C}$ for $20 \mathrm{~min}$. The reaction of grafting was carried out in dry state at $180^{\circ} \mathrm{C}$ for $30 \mathrm{~min}$. The treated and cured textile materials were rinsed with warm distilled water and dried at room temperature to constant mass. The weight gain of the finished fabrics was measured (DIN 53814) to yield the efficiency of the treatment. The samples were dried for $4 \mathrm{~h}$ at $105^{\circ} \mathrm{C}$ and weighed before and after finishing and rinsing. Depending on the initial concentrations of all reactants (maintaining the concentration of $\alpha$-cellulose constant) the weight gain varies and is reported in Table 1 . As one can observe in Table 1 , the grafting of $\beta-C D$ to the cellulose backbone depends on both the initial concentration of $C D$ and that of cross linker (tetracarboxylic acid, BTCA); an increase in the concentrations of both results in a weight gain to cellulose. This increase in cellulose weight is related mainly to the amount of $\beta-C D$ grafted to the cellulose backbone; the influence of the BTCA mass can be neglected due to low molecular mass of BTCA compared to the molecular mass of $\beta-C D$. For IR spectroscopy analysis the treated $\alpha$-cellulose was soaked in alkaline solution of $0.1 \mathrm{M} \mathrm{NaOH}$ (to convert all the remained carboxylic acid groups to carboxylate groups), rinsed in warm distilled water, dried and weighed.

\subsection{Techniques}

IR spectroscopy was carried out with a Perkin-Elmer Fourier Transform infrared (FTIR) spectrophotometer with a Golden Gate attenuated total reflection (ATR) attachment with a diamante crystal and in a Nicolet 6700 FTIR spectrometer.

Thermal gravimetric analysis (TGA) was performed on a TGA/SDTA 851 Mettler Toledo thermal analyzer. Samples were heated from room temperature up to $600^{\circ} \mathrm{C}$ at $10^{\circ} \mathrm{C} \mathrm{min}^{-1}$ under a nitrogen atmosphere $\left(50 \mathrm{~cm}^{3} \mathrm{~min}^{-1}\right)$.

Polarized optical microscopy A Linkam LTS 120 microscope equipped with a Q imaging (Qicam) Fast 1394 camera was used to observe the cellulose fiber dissolution in the acid solvent. Samples were kept between coverslips and illuminated with linearly polarized light and analyzed through a crossed polarizer. Images were captured and analyzed using Qcapture software.

${ }^{13} \mathrm{C}$ Solid state NMR, Cross Polarization Magic Angle Spinning Nuclear Magnetic Resonance (CPMAS NMR), measurements were performed on a Bruker Avance II spectrometer (Karlsruhe, Germany) operating at $300.13 \mathrm{MHz}$ regarding ${ }^{1} \mathrm{H}$. Spinning speed of the samples was set to $5 \mathrm{kHz}$ at the magic angle, at room 


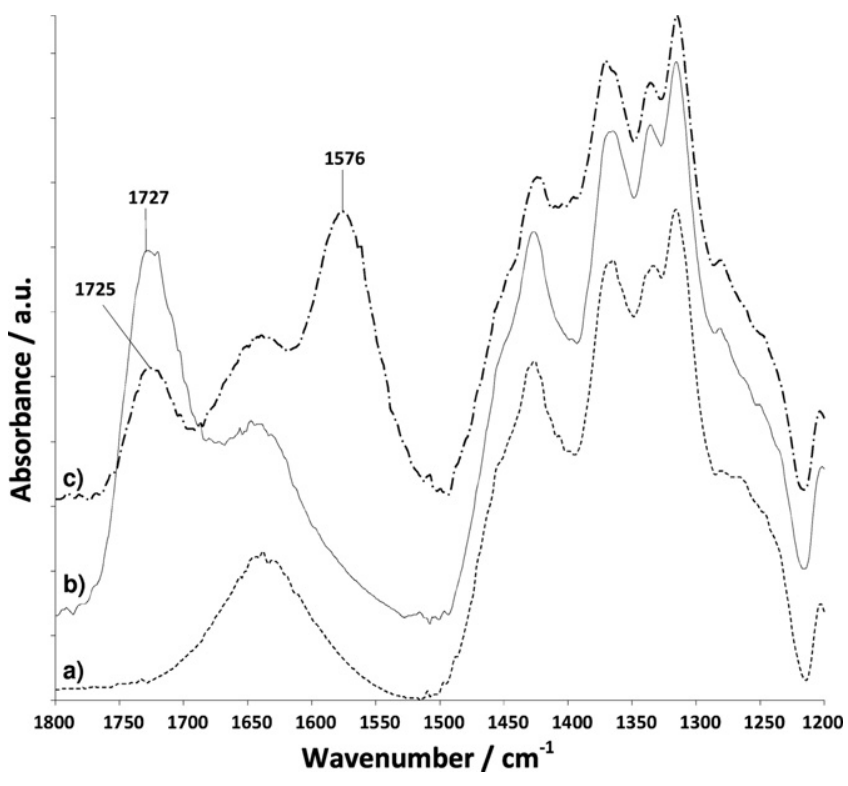

Fig. 1. Infrared spectra of native $\alpha$-cellulose (spectrum a), CD-modified cellulose (spectrum b) and CD-modified cellulose after alkaline treatment (spectrum c) at room temperature.

temperature. ${ }^{13} \mathrm{C}$ signal was collected after ${ }^{1} \mathrm{H}$ decoupling (Bennett, Rienstra, Auger, Lakshmi, \& Griffin, 1995) and the ${ }^{1} \mathrm{H}$ and ${ }^{13} \mathrm{C}$ radiofrequency field strengths were set to give $90^{\circ}$ pulse durations around 2.5 and $4 \mu \mathrm{s}$, respectively. $\mathrm{CP}$ experiments were conducted with a contact pulse of $900 \mu \mathrm{s}$.

\section{Results and discussion}

\subsection{Characterization of the unmodified and modified polymer}

In Fig. 1, FTIR spectra of unmodified native $\alpha$-cellulose (spectrum a), modified $\alpha$-cellulose with $\beta$-CD grafted to the cellulose backbone by means of BTCA (spectrum b) and modified $\alpha$-cellulose cellulose after-treated in alkaline solution (spectrum c) are presented.

Only one set of spectra is shown (all the others are qualitatively the same). A new vibration band at $1727 \mathrm{~cm}^{-1}$ (spectrum b, modified polymer) can be observed and assigned to a $\mathrm{C}=\mathrm{O}$ stretching vibration mode characteristic for an ester bond. This suggests that the carboxylic acid forms ester bonds with the hydroxyl groups from CD and $\alpha$-cellulose. When cellulose was treated with BTCA and $\beta-C D$, esterification between BTCA, $\beta-C D$ and the hydroxyl groups of cellulose occurred at elevated temperature; Fig. 1 shows the appearance of a new band (spectrum b) after such treatment. We should note that the carbonyls retained in the cellulose may exist in three forms: as esters, carboxylic acids or carboxylate anions. In IR spectroscopy the band due to an ester carbonyl group appears around $1727 \mathrm{~cm}^{-1}$ but the band due to a carbonyl group in a carboxylic acid also appears in the same region meaning that in the BTCA $/ \beta-C D$-finished cellulose the band for the ester group cannot be resolved without further treatment. However, we can separate the ester carbonyl band from the acid carbonyl band by converting the carboxylic acid group to carboxylate anions in an alkaline solution as exemplified in Fig. 2. As we can see in Fig. 1 (spectrum c), in the FTIR spectrum of the after-treated BTCA/ $\beta-C D$ finished cellulose a new band at $1576 \mathrm{~cm}^{-1}$ arises, thus confirming the existence of free carboxylates while maintaining the band at $1727 \mathrm{~cm}^{-1}$ band (slightly lower intensity) attributed to the ester bond between BTCA, CD and cellulose (Fig. 2).<smiles>COC(=O)CC(C(=O)O)C(CC(=O)OCl)C(=O)O</smiles><smiles>O=C(CC(CC(=O)O[Na])C(CC(=O)O[Na])C(=O)[O-])O[Na]</smiles>

Fig. 2. Conversion of carboxylic acid groups to carboxylate anions after alkaline treatment of the cellulose- $\mathrm{CD}$ modified polymer.

The esterification has also been confirmed by ${ }^{13} \mathrm{C}$-solid state CPMAS NMR (Fig. 3) where a new peak at 173 ppm, for the modified sample, can be assigned to the $\alpha$-carbon in an ester bond. All the other signals can easily be assigned to the remaining carbons of the glucopyranose units (Goud et al., 2012). By changing spinning frequencies of the sample it was confirmed that the new peak is not an artifact, but a consequence of the new-formed chemical bond. Thus, the proposed mechanism consists of the crosslinking of cellulosic fibers by BTCA in the presence of phosphorous catalyst. The reaction occurs through dehydration below the curing temperature of the polycarboxylic acid, which yields a cyclic anhydride intermediate that readily reacts with the hydroxyl groups of cellulose by esterification. Then, two of the remaining carboxylic groups of the reacted polycarboxylic acid can form a second anhydride that can also react with another cellulosic unit from another macromolecular chain (schematic reaction scheme in Fig. 4). Note that the formation of cyclic anhydride intermediates has been suggested to occur when a polycarboxylic acid is used as the esterification agent in cotton. Due to the obvious similarities in chemical structure (cotton is essentially pure cellulose) we therefore believe the same mechanism can be proposed here. Thermal gravimetric analysis of all samples (unmodified, Ref., and modified samples, A, B and $\mathrm{C}$ - see Table 1 for composition details) has been undertaken and thermograms are shown in Fig. 5. Two degradation steps can be found up to $600^{\circ} \mathrm{C}$; the first one at a temperature around $100^{\circ} \mathrm{C}$ is due to the loss of moisture, whilst the second one is due to the start of decomposition of the glucose units of cellulose and, probably, CD as well. Table 2 shows the temperatures at the maximum degradation rate $\left(T_{\max }\right)$ of functionalized $\alpha$-cellulose, as determined from the maximum of DTG curves. Two important features are noticed: (a) the incorporation of $C D$ leads to a decrease in the $T_{\max }$, which can be understood from the presence of $\mathrm{CD}$ (with a degradation temperature around $325^{\circ} \mathrm{C}$, Ehen, Giordano, Sztatisz, Jicsinszky, \& Novak, 2005) and/or by an increase in the disorder of cellulose molecular packing; and (b) an increase of the amount of CD introduced leads to a decrease of the weight loss on the main degradation transition. We will further discuss this observation after discussing the solubility results.

\subsection{Dissolution of the unmodified and modified cellulose}

An important issue related with cellulose and its derivatives is solubility, a controversial and unsettled issue (Lindman et al., 2010); it should be expected that with an incorporation of CD, the solubility in water should increase both due to an increase of hydrophilic area (exterior of CD is hydrophilic) and to a decrease of the association between cellulose molecules by steric hindrance. Several solvents were initially used to promote cellulose dissolution, namely: $\mathrm{ZnCl}_{2}$ (Fischer, Leipner, Thummler, Brendler, \& Peters, 2003), $\mathrm{NaOH}$ (20\% and 9\%) (Isogai \& Atalla, 1998; Navard \& Le Moigne, 2010) and "superphosphoric" acid ( $\mathrm{P}_{2} \mathrm{O}_{5}$ content of 74\%) (Boerstoel, Maatman, Westerink, \& Koenders, 2001; Northolt et al., 

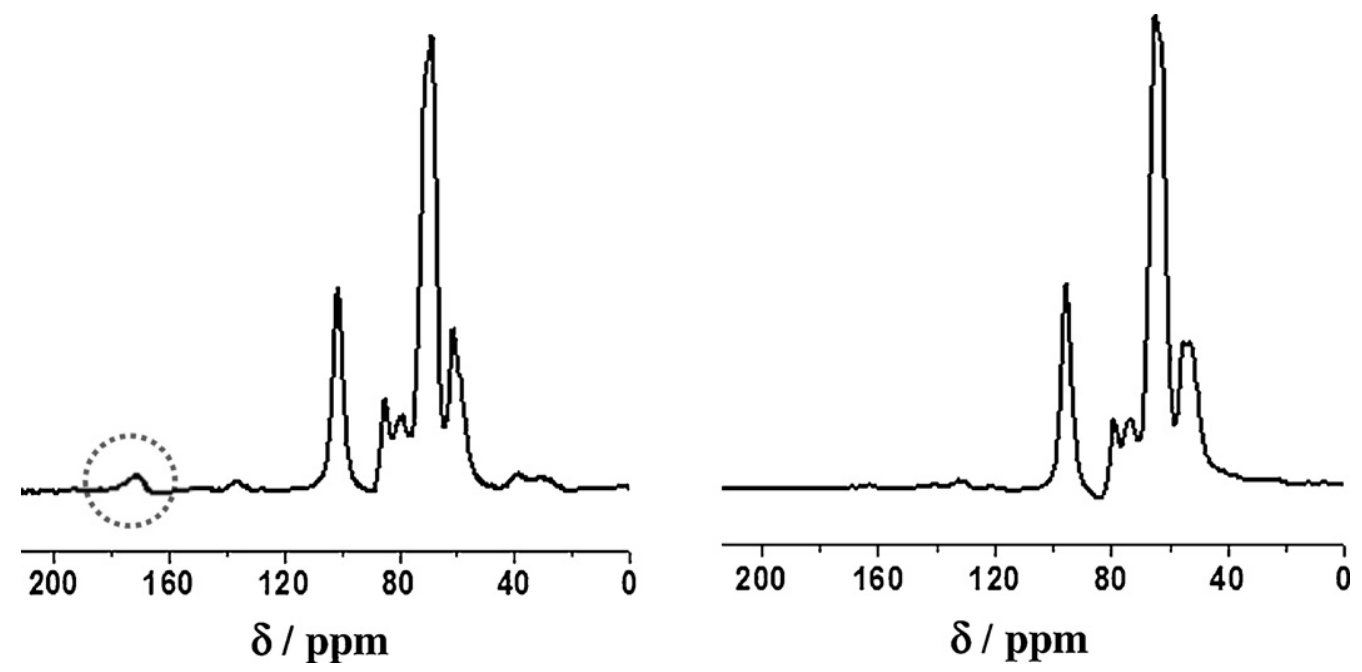

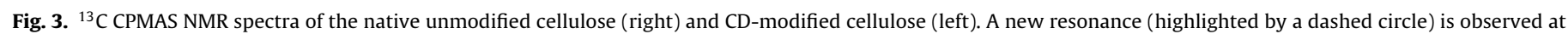
$173 \mathrm{ppm}$ and can be attributed to a carbon in an ester bond.

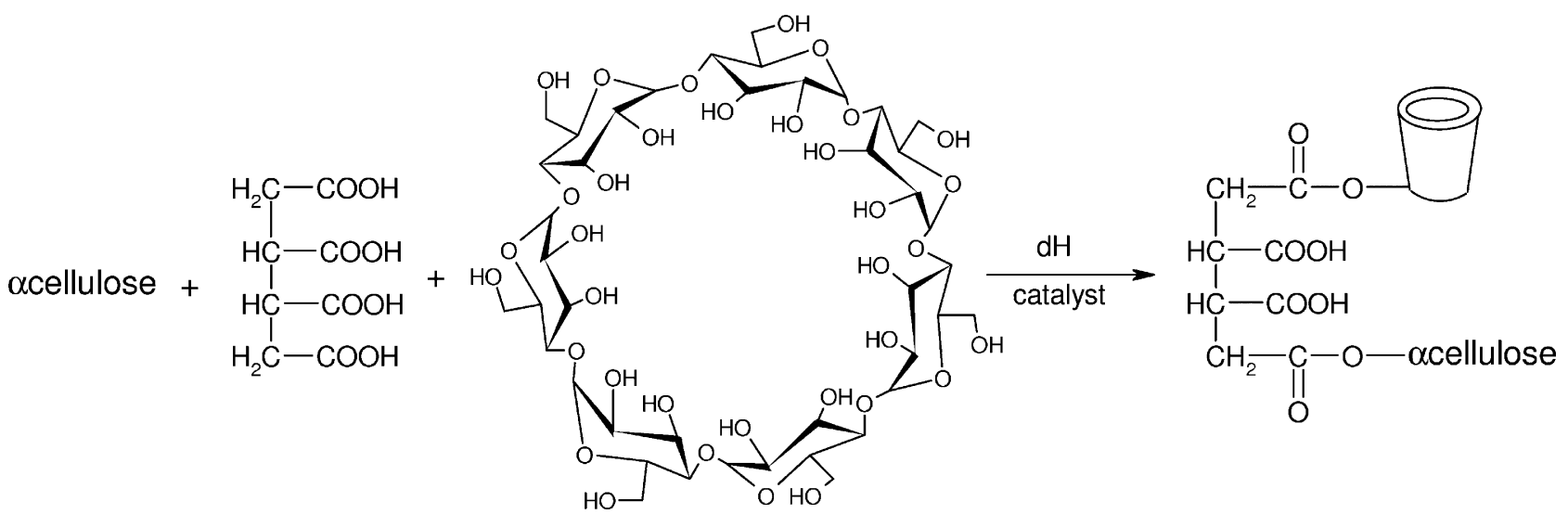

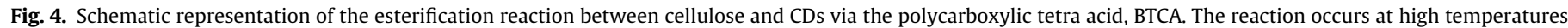
in the presence of catalyst.

2001). Generally, the procedure was the same for all solvents; at room temperature, a certain amount of water was added to cellulose (hydration step). Afterwards, the solvent was added to the

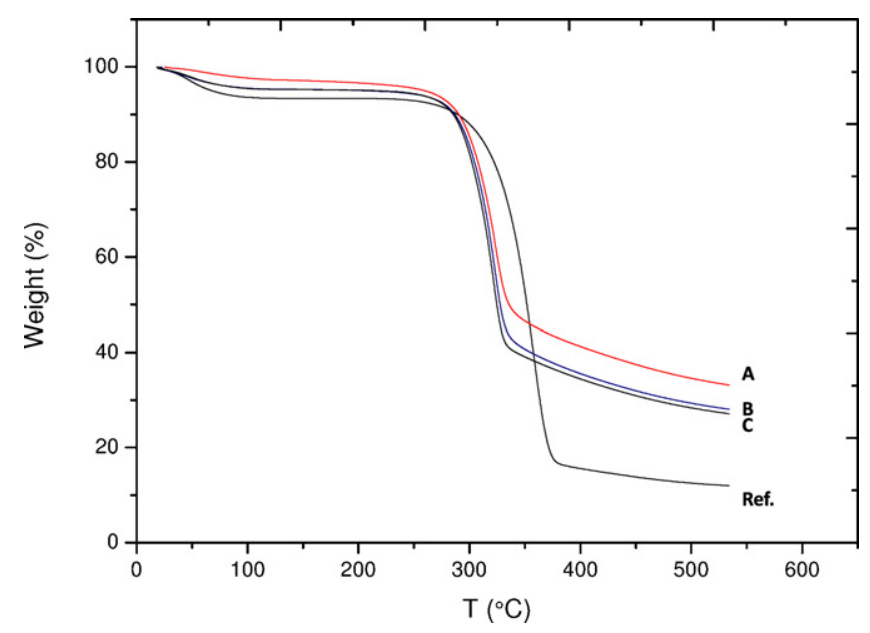

Fig. 5. Thermograms of the unmodified native cellulose (Ref.) and CD-modified cellulose (A, B and C - see Table 1 for details). Two main transitions can be observed. $\mathrm{T}_{50}$ is estimated and reported in Table 2. cellulose and the sample vigorously mixed. Qualitatively the same features were observed in all the solvents used and thus we will report only the results obtained with "superphosphoric" acid. Fig. 6 shows the typical phase behavior of the unmodified and modified samples; in the case of the unmodified cellulose, dissolution was fully succeeded in the acid solvent. On the other hand, regardless of the conditions (cellulose concentration, temperature and mixing conditions) phase separation was always observed when using the cellulose modified with CDs. The solubilization of cellulose in polyphosphoric acid/water mixture was also followed by polarized optical microscopy. As can be inferred from Fig. 7 one observes full dissolution of native unmodified cellulose in the acidic solvent. However, in the case of CD-modified cellulose, although some degree of swelling is observed, insoluble material is clearly present.

Table 2

Degradation temperature and weight loss at $590^{\circ} \mathrm{C}$ of different $\alpha$-cellulose samples. The sample "Ref." is the native unmodified $\alpha$-cellulose while all the others are modified products (see Table 1 ).

\begin{tabular}{lll}
\hline Sample & $T_{\max }\left({ }^{\circ} \mathrm{C}\right)$ & Weight loss (\%) \\
\hline Ref. & 347 & 88.6 \\
A & 317 & 68.6 \\
B & 316 & 73.5 \\
C & 314 & 74.4 \\
\hline
\end{tabular}



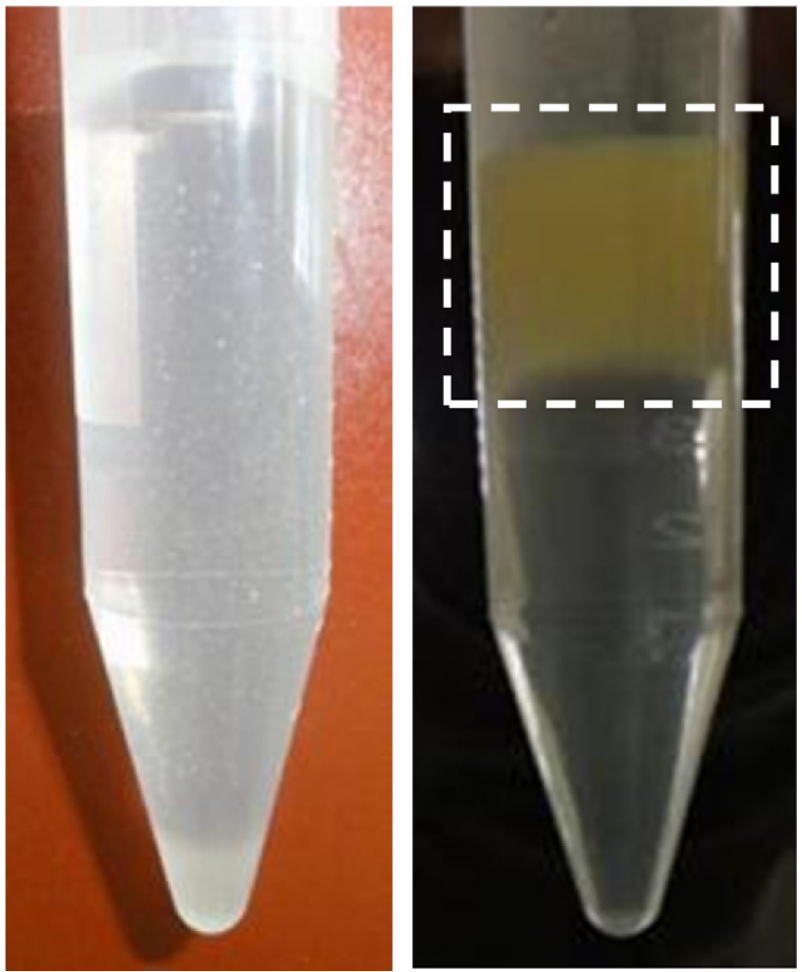

Fig. 6. Dissolution of $2.5 \%$ cellulose in a "superphosphoric" acid $/ \mathrm{H}_{2} \mathrm{O}$ solvent $(74 \%$ $\mathrm{P}_{2} \mathrm{O}_{5}$ ). On the left side (unmodified native cellulose, Ref.) one can observe a fully dissolved transparent sample while on the right side (CD-modified cellulose, A) a clear phase separation is highlighted by the dashed square.

\subsection{In(solubility): possible explanation}

The full dissolution of the modified cellulose is clearly not an easy task. As mentioned above, one would expect an increase of polarity and hydrophilicity with the chemical modification which should, in principle, lead to an easier dissolution of modified cellulose in aqueous solvents. In fact, this is a scenario normally found when cellulose is modified with other groups (for instance, methyl cellulose, hydroxyethyl cellulose and methylhydroxyethyl cellulose are soluble in water) (Lindman et al., 2010). However, when CDs were chemically attached to cellulose the modified polymer became less soluble.

Fig. 8 illustrates two possible scenarios of the synthesis outcome. On the left side, one considers a hypothetic synthesis from which the formation of individual cellulose molecules grafted with $n$ CDs would be the main product. In this case, one would expect an easier dissolution of cellulose fibers (arguments exposed above). However, another scenario can also be considered (Fig. 8, right). The cyclodextrin itself could act as a bridge connecting different cellulose fibers forming a three-dimensional chemical network making the solvent access, swelling and dissolution more restricted. Since the esterification reaction occurs in dry conditions, there is a clear excess of cellulose and thus the CD-cellulose interactions occur in an unselective way. Moreover, due to the CD size, high surface area and available hydroxyl groups, the possibility of one $\mathrm{CD}$ grafting more than one cellulose fiber is plausible (through the BTCA esterification). The TGA analysis also seems to support the formation of a complex three-dimensional network of cellulose fibers chemically linked by CDs. Such a network would be less resistant to solvent penetration as a consequence of the higher mobility of cellulose skeleton provided by the void arised by the presence of CDs among the cellulose structure, as suggested by the significant decrease on the $T_{\max }$ of functionalized $\alpha$-cellulose. As one can see in Fig. 5 the weight lost for the modified samples was considerably lower than in the case of the native unmodified cellulose samples. We do not exclude the possibility of cellulose being cross-linked only with BTCA without the presence of CDs. Indeed, one might have a mixture of different crosslinked products (cellulose-BTCA-cellulose; cellulose-BTCA-CD; cellulose-BTCA-CD-BTCA-cellulose) as exemplified in Fig. 8.
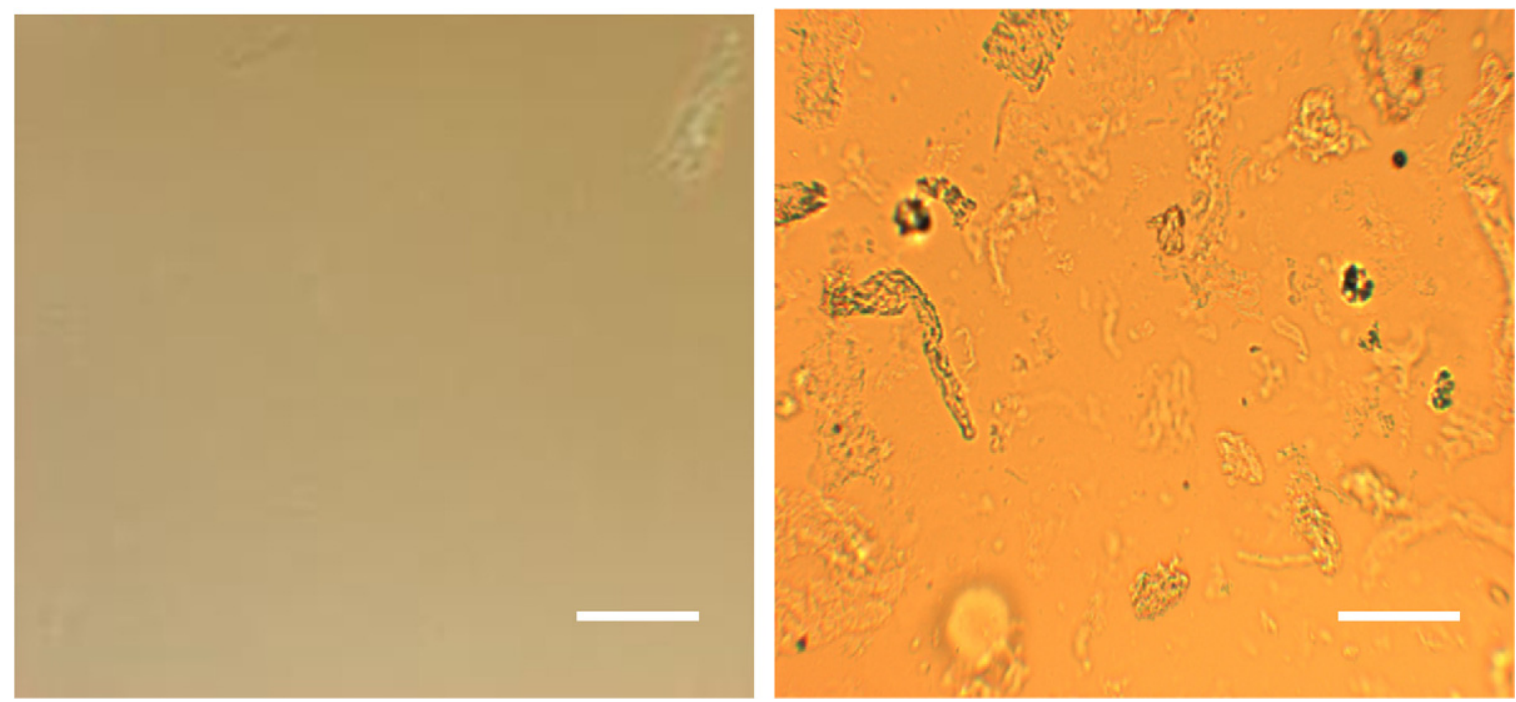

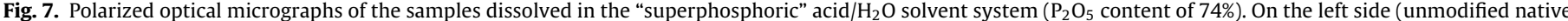

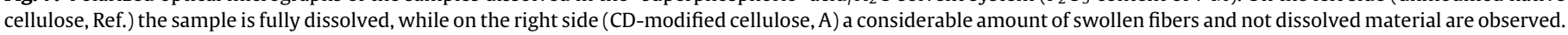
The white bar represents $5 \mu \mathrm{m}$. 


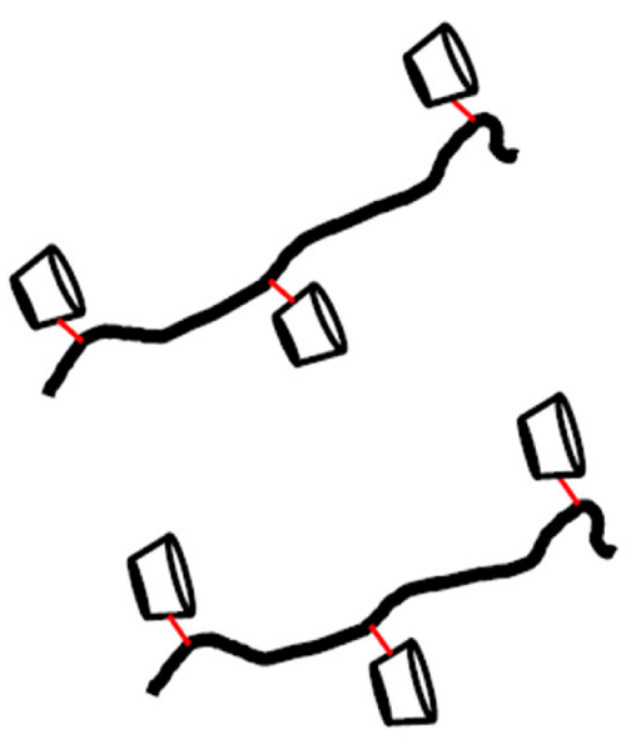

A

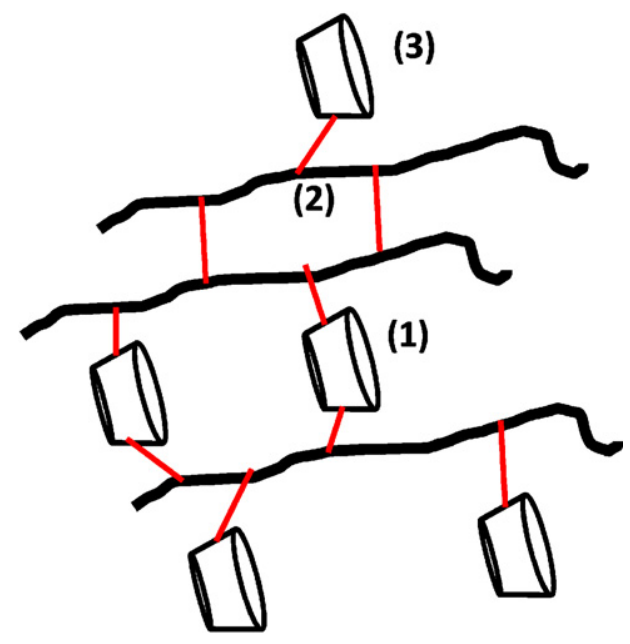

B

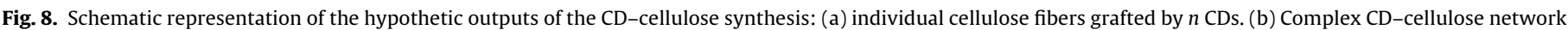

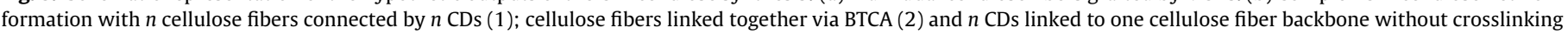
a second fiber (3).

\section{Conclusions}

Cellulose (in)solubility and functionalization is still a hot topic. Here we have revisited a simple synthesis procedure and the parameters that control it. It was possible to modify cellulose with cyclodextrins using a simple polycarboxylic acid as cross-linking agent. Solid state NMR and FTIR showed that ester bonds are formed between cellulose and cyclodextrins. The chemical modification of cellulose was expected to make the natural polymer more soluble (disruption of crystalline structure and making cellulose more polar). Unexpectedly, however, it was not possible to fully dissolve the $\mathrm{CD}$-cellulose complex probably due to a complex network formation where BTCA can cross-link cellulose molecules and/or single CDs can cross-link several cellulose molecules. As a consequence of this network formation full dissolution is prevented as observed by polarized light microscopy and the thermal degradation of the modified polymers is less extensive and occurs with considerable less weight loss.

\section{Acknowledgment}

We acknowledge Fundação para a Ciência e Tecnologia (FCT) for the grant conceded for a Portugal-Slovenia project and for a post-doctoral fellowship (BM: SFRH/BPD/74540/2010). The Ministry of Education, Science, Culture and Sport (MIZKS) and Södra are acknowledge for financial support.

\section{References}

Akasaka, M., Sawai, Y., Iwase, K., \& Moriishi, H. (1992). EP 488294 Akasaka, M., Shibata, T., \& Ochia, H. (1991). Jpn. Kokai Tokkyo Koho 03059178 Bennett, A. E., Rienstra, C. M., Auger, M., Lakshmi, K. V., \& Griffin, R. G. (1995). Heteronuclear decoupling in rotating solids. Journal of Chemical Physics, 103(16) 6951-6958.

Boerstoel, H., Maatman, H., Westerink, J. B., \& Koenders, B. M. (2001). Liquid crystalline solutions of cellulose in phosphoric acid. Polymer, 42(17), 7371-7379.

Buschman, H. J., Denter, U., Knittel, D., \& Schollmeyer, E. (1998). The use of cyclodextrins in textile processes - An overview. Journal of the Textile Institute, 89(3), 554-561.

Buschmann, H. J. (2001). Cosmetic textiles: Clothes with a skin care action. Cossma, 2(7), 38.
Buschmann, H. J., Knittel, D., Beermann, K., \& Schollmeyer, E. (2001). Cyclodextrins and textiles. Nachrichten aus Chemie, 49(5), 620.

Buschmann, H. J., Knittel, D., \& Schollmeyer, E. (1991). Resin finishing of cotton in the presence of cyclodextrins for depositing fragrances. Melliand Textilberichte International Textile Reports, 72(3), 198.

Buschmann, H. J., Knittel, D., \& Schollmeyer, E. (2001). Use of cyclodextrins for improving waste air washers. Melliand Textilberichte International Textile Reports, 82(5), 368

Del Valle, M. E. M. (2004). Process Biochemistry, 39, 1033.

Denter, U., Buschmann, H. J., Knittel, D., \& Schollmeyer, E. (1997). Modification of fiber surfaces by permanent fixation of supramolecular components. 2. Cyclodextrines. Angewandte Makromolekulare Chemie, 248, 165-188.

Denter, U., \& Schollmeyer, E. (1996). Surface modification of synthetic and natural fibres by fixation of cyclodextrin derivatives. Journal of Inclusion Phenomena and Molecular Recognition in Chemistry, 25(1-3), 197-202.

Ehen, Z., Giordano, F., Sztatisz, J., Jicsinszky, L., \& Novak, C. (2005). Thermal characterization of natural and modified cyclodextrins using TG-MS combined technique. Journal of Thermal Analysis and Calorimetry, 80(2), 419-424.

Fischer, S., Leipner, H., Thummler, K., Brendler, E., \& Peters, J. (2003). Inorganic molten salts as solvents for cellulose. Cellulose, 10(3), 227-236.

Fujimura, T. (1985). Jpn. Kokai Tokkyo Koho 60259648.

Gagliardi, D. D., \& Shippee, F. B. (1963). American Dyestuff Reporter, 15, 74.

Goud, N. R., Gangavaram, S., Suresh, K., Pal, S., Manjunatha, S. G., Nambiar, S., et al. (2012). Journal of Pharmaceutical Sciences, 101(2), 664-680.

Guyot, M., Fawaz, F., Bildet, J., Bonini, F., \& Lagueny, A. M. (1995). Physicochemical characterization and dissolution of norfloxacin/cyclodextrin inclusioncompounds and peg solid dispersions. International Journal of Pharmaceutics, 123(1), 53-63.

Isogai, A., \& Atalla, R. H. (1998). Dissolution of cellulose in aqueous $\mathrm{NaOH}$ solutions. Cellulose, 5(4), 309-319.

Li, S., \& Purdy, W. C. (1992). Cyclodextrins and their applications in analyticalchemistry. Chemical Reviews, 92(6), 1457-1470.

Lindman, B., Karlstrom, G., \& Stigsson, L. (2010). On the mechanism of dissolution of cellulose. Journal of Molecular Liquids, 156(1), 76-81.

Lis, S., Elbanowski, M., Makowska, B., \& Hnatejko, Z. (2002). Energy transfer in solution of lanthanide complexes. Journal of Photochemistry and Photobiology A: Chemistry, 150(1-3), 233-247.

Martel, B., Ducoroy, L., Bacquet, B., \& Morcellet, M. (2007). Ion exchange textiles from the finishing of PET fabrics with cyclodextrins and citric acid for the sorption of metallic cations in water. Journal of Inclusion Phenomena and Macrocyclic Chemistry, 57(1-4), 271-277.

Martel, B., Ducoroy, L., Bacquet, M., \& Morcellet, M. (2007). Cation exchange finishing of nonwoven polyester with polycarboxylic acids and cyclodextrins. Journal of Applied Polymer Science, 103(6), 3730-3738.

Martel, B., Morcellet, M., Ruffin, D., Ducoroy, L., \& Weltrowski, M. (2002). Finishing of polyester fabrics with cyclodextrins and polycarboxylic acids as crosslinking agents. Journal of Inclusion Phenomena and Macrocyclic Chemistry, 44(1-4), 443-446.

Martel, B., Ruffin, D., Weltrowski, M., Lekchiri, Y., \& Morcellet, M. (2005). Watersoluble polvmers and gels from the polycondensation between cyclodextrins and poly(carboxylic acid)s: A study of the preparation parameters. Journal of Applied Polymer Science, 97(2), 433-442. 
Martel, B., Weltrowski, M., Ruffin, D., \& Morcellet, M. (2002). Polycarboxylic acids as crosslinking agents for grafting cyclodextrins onto cotton and wool fabrics: Study of the process parameters. Journal of Applied Polymer Science, 83(7), 1449-1456.

Morris, N. M., Catalano, E. A., \& Andrews, B. A. K. (1995). FT-IR determination of degree of esterification in polycarboxylic acid cross-link finishing of cotton. Cellulose, 2(1), 31-39.

Murthy, C. N., \& Shown, I. (2008). Synthesis and characterization of linear water-soluble gamma-cyclodextrin based polymers as drug carrier systems. Supramolecular Chemistry, 20(6), 573-578.

Murthy, C. N., \& Shown, I. (2009). Grafting of cotton fiber by water-soluble cyclodextrin-based polymer. Journal of Applied Polymer Science, 111(4), 2056-2061.

Natoli, M., Pagliero, C., Trotta, F., \& Drioli, E.(1997). A study of catalytic p-cyclodextrin carbonate membrane reactor performance in PNPA hydrolysis. Journal of Molecular Catalysis A: Chemical, 121(2-3), 179-186.

Navard, P., \& Le Moigne, N. (2010). Dissolution mechanisms of wood cellulose fibres in $\mathrm{NaOH}$-water. Cellulose, 17(1), 31-45.

Northolt, M. G., Boerstoel, H., Maatman, H., Huisman, R., Veurink, J., \& Elzerman, H. (2001). The structure and properties of cellulose fibres spun from an anisotropic phosphoric acid solution. Polymer, 42(19), 8249-8264.

Poulakis, K., Buschmann, H. J., \& Schollmeyer, E. (1992). German Pat DE 4035378 A1.

Poulakis, K., Buschmann, H. J., \& Schollmeyer, E. (2002). Ger. Offen. 4035378 (1992): WO 2002046520.

Reuscher, H., Hirsenkorn, R., \& Haas, W. (1995). EP 0697415A1.

Ritter, W., Delney, J., Volz, W., \& Kerr, I. (2002). DE 10101294.

Rowland, S. P. (1968). Textile Research Journal, 38, 643.
Rowland, S. P., Welch, C. M., Brannan, M. A. F., \& Gallagher, D. M. (1967). Textile Research Journal, 37, 933.

Saenger, W. R., Jacob, J., Gessler, K., Steiner, T., Hoffmann, D., Sanbe, H., et al. (1998) Structures of the common cyclodextrins and their larger analogues - Beyond the doughnut. Chemical Reviews, 98(5), 1787-1802.

Savarino, P., Viscardi, G., Quagliotto, P., Montoneri, E., \& Barni, E. (1999). Reactivity and effects of cyclodextrins in textile dyeing. Dyes and Pigments, 42(2), 143-147.

Szejtli, J. (2003). Cyclodextrins in the textile industry. Starch-Starke, 55(5), 191-196

Szejtli, J., Zsadon, E., Fenyvesi, E., Horvath, K., \& Tudos, F. (1980). Adsorbent cellulosecyclodextrin-epoxide reaction prod. - capable of forming inclusion complexes (p. 7). Chinoin Gyogyszer Es Vegyeszeti (Chin).

Uekama, K., Hirayama, F., \& Irie, T. (1998). Cyclodextrin drug carrier systems. Chemical Reviews, 98(5), 2045-2076.

Utsuki, T., Brem, H., Pitha, J., Loftsson, T., Kristmundsdottir, T., Tyler, B. M., et al. (1996). Potentiation of anticancer effects of microencapsulated carboplatin by hydroxypropyl alpha-cyclodextrin. Journal of Controlled Release, 40(3), 251-260.

Vogtle, F. (Ed.). (1991). Supramolecular chemistry: An introduction. New York: Wiley.

Voncina, B., \& Le Marechal, A. M. (2005). Grafting of cotton with beta-cyclodextrin via poly(carboxylic acid). Journal of Applied Polymer Science, 96(4), 1323-1328.

Voncina, B., \& Majcen, N. (2004). Application of cyclodextrin for medical and hygienic textiles. Tekstil, 53(1), 1-9.

Voncina, B., Vivod, V., \& Jausovec, D. (2007). beta-Cyclodextrin as retarding reagent in polyacrylonitrile dyeing. Dyes and Pigments, 74(3), 642-646.

Welch, C. M. (1988). Textile Research Journal, 58, 480.

Yamamoto, K., \& Saeki, T. Jpn. Kokai Tokkyo Koho 09228144 (1997) and 10007591 (1998).

Zelinski, T., Liese, A., Wandrey, C., \& Kula, M. R. (1999). Asymmetric reductions in aqueous media: Enzymatic synthesis in cyclodextrin containing buffers. Tetrahedron: Asymmetry, 10(9), 1681-1687. 